\title{
Electronic Properties of Nanopore Edges of Ferromagnetic Graphene Nanomeshes at High Carrier Densities under Ionic-Liquid Gating
}

\author{
Taijyu Hashimoto, Syota Kamikawa, Yuko Yagi, Junji Haruyama*
}

Faculty of Science and Engineering, Aoyama Gakuin University, Sagamihara, Japan.

Email: J-haru@ee.aoyama.ac.jp

Received November 21 ${ }^{\text {st }}$, 2013; revised December 20 ${ }^{\text {th }}$, 2013; accepted December $31^{\text {st }}, 2013$

Copyright (C 2014 Taijyu Hashimoto et al. This is an open access article distributed under the Creative Commons Attribution License, which permits unrestricted use, distribution, and reproduction in any medium, provided the original work is properly cited. In accordance of the Creative Commons Attribution License all Copyrights (C) 2014 are reserved for SCIRP and the owner of the intellectual property Taijyu Hashimoto et al. All Copyright (C) 2014 are guarded by law and by SCIRP as a guardian.

\section{ABSTRACT}

Graphene edges with a zigzag-type atomic structure can theoretically produce spontaneous spin polarization despite being a critical-metal-free material. We have demonstrated this in graphene nanomeshes (GNMs) with honeycomb-like arrays of low-defect hexagonal nanopores by observing room-temperature ferromagnetism and spin-based phenomena arising from the zigzag-pore edges. Here, we apply extremely high electric fields to the ferromagnetic (FM) GNMs using an ionic-liquid gate. A large on/off-ratio for hole current is observed for even small applied ionic-liquid gate voltages $\left(V_{\text {ig }}\right)$. Observations of the magnetoresistance behavior reveal high carrier densities of $\sim 10^{13} \mathrm{~cm}^{-2}$ at large $V_{\text {ig }}$ values. We find a maximum conductance peak in the high $-V_{\text {ig }}$ region and its separation into two peaks upon applying a side-gate (in-plane external) voltage $\left(V_{e x}\right)$. It is discussed that localized edge- $\pi$ band with excess-density electrons induced by $V_{\text {ig }}$ and its spin splitting for majority and minority of spins by $V_{\text {ex }}$ (half-metallicity model) lead to these phenomena. The results must realize critical-element-free novel spintronic devices.

\section{KEYWORDS}

Graphene; Nanomesh; Edge; Spin Polarization; Magnetism; Ionic-Liquid Gate; High Electric Fields; Rare-Metal-Free Spintronics

\section{Introduction}

The presence of spontaneously spin-polarized electrons has been theoretically reported at zigzag-atomic structure of edges of graphenes with flat energy bands [1-3]. We experimentally reported it at the hydrogen $(H)$-terminated pore edges of low-defect graphene nanomeshes (GNMs: Figure 1(a)) [1-15], which were fabricated using non-lithographic method (i.e., nanoporous alumina templates as etching masks [11]), by observing the ferromagnetism (i.e., ferromagnetic GNMs (FM-FNMs)) [1-9], the distribution of the polarized edge spins by magnetic force microscope [10], and some spin-based phenomena [10-15]. The spin polarization appeared because of the low-defect zigzag atomic structure of the

\footnotetext{
${ }^{*}$ Corresponding author.
}

pore edges [1-7] formed by edge reconstruction caused by critical-temperature annealing [12-14] and the assembly of narrow interpore regions (e.g., $10 \sim 20 \mathrm{~nm}$ width) corresponding to $H$-terminated zigzag graphene nanoribbons (GNRs: one-dimensional strip lines of graphene with edges on both longitudinal sides; Figure 1(a) $[8,9]$ ). A large ensemble of GNRs in a GNM enabled the emergence of large-magnitude ferromagnetism even at room temperature.

On the other hand, recently, several studies have reported achieving extremely high carrier densities on material surfaces (e.g., observing the transition from insulators to superconductors and carrier filling in higher energy bands) using electrolytic gates (e.g., ionic liquids and ionic solid-polymers: Figures 1(c) and (d)) [16-23]. The method enables the realization of a drop in the electric 


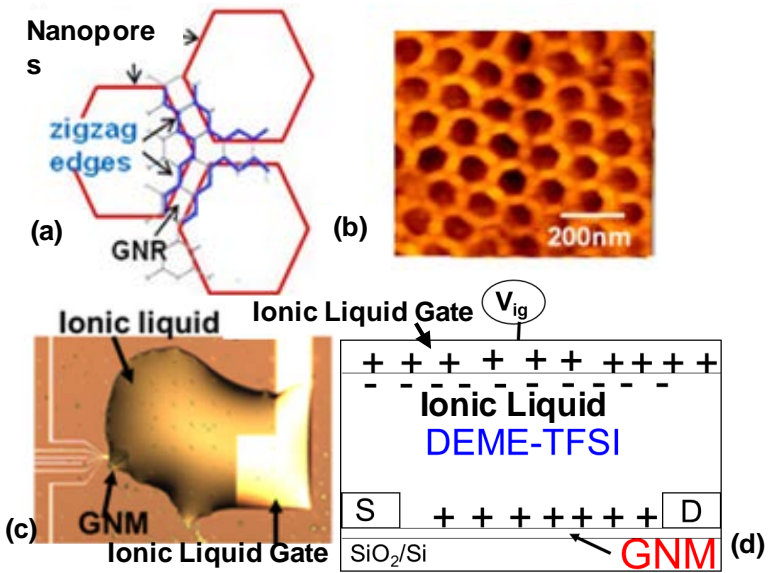

Figure 1. Sample structures (a) Schematic top-view and (b) atomic force microscope image of hydrogen $(\mathrm{H})$-terminated zigzag GNMs, which gives a honeycomb-like array of lowdefect hexagonal nanopores. The mean pore diameter and interpore distance are $\sim 80 \mathrm{~nm}$ and $\sim 20 \mathrm{~nm}$, respectively. The interpore regions can be $\boldsymbol{H}$-terminated graphene nanoribbons (GNRs) with a width of $\sim 20 \mathrm{~nm}$ and, thus, the GNM can be a large ensemble of $\boldsymbol{H}$-terminated-zigzag GNRs, resulting in the appearance of large ferromagnetism. (c),(d) Hall-measurement pattern of the $~ 5$-layer ferromagnetic (FM) GNM with an ionic-liquid gate using DEME-TFSI [19]. Side-gate electrode, which is located within $\sim 500 \mu \mathrm{m}$ distance from the GNM and is not covered by ionic liquid, was used for applying $V_{\text {ex }}$.

field only at the interface of the material surface (e.g., only for a thickness of $1 \mathrm{~nm}$ ) via the formation of a Debye layer (Figure $\mathbf{1}(\mathrm{d})$ ), thereby resulting in the concentration of an extremely high electric field at the surface. The method has been also applied to graphenes. For example, high carrier densities obtained by this method ( $\gg$ $10^{14} \mathrm{~cm}^{-2}$ ) have revealed the presence of the quantum distribution of two-dimensional (2D) acoustic phonons in graphenes. Further, the filling of the high-energy subbands has been observed along with high electron mobility in bilayer graphenes $[16,17]$. These results indicate the importance of the high carrier densities observed in graphenes (edges) in the light of examining novel electronic states and physical phenomena.

Indeed, a variety of spin-related phenomena have been predicted based on the study of the edge spins of graphene [6,24-28]. For instance, half-metallicity was predicted in GNRs with antiferromagnetic (AF) spin alignment at two zigzag edges [6]. In such a GNR, the gap for the spins with the same moment (e.g., up spins) between both edges disappears upon applying in-plane external electric fields $\left(\mathrm{V}_{\mathrm{ex}}\right)$, while the gap for the opposite-moment spins (e.g., down spins) increases. The occurrence of the (quantum) spin Hall effect was also predicted by resolving the double degeneration of edge spin bands (e.g., by introducing spin-orbit interactions) and control- ling two spins with opposite moments existing in two different bands by applying electric fields $[25,26]$. In this light, a large spin current was experimentally observed using Zeeman splitting in high-quality bulk graphenes positioned on h-boron-nitride (BN) [27]. We have also reported possible spin-based phenomena arising from the pore edges of 5-layer FM-GNMs [28].

In the present study, we fabricate an ionic-liquid gate on 5-layer FM-GNMs with polarized spins at the pore edges, and we observe the changes in the pore-edge electronic and spintronic states induced by the applied ionic-liquid gate voltage $\mathrm{V}_{\text {ig }}$ and also the side-gate voltage $\mathrm{V}_{\mathrm{ex}}$ via an electrode not connected to the ionic liquid.

\section{Experimental Results and Discussions}

\subsection{Sample Fabrication}

Figure 1 shows the top-view schematic (a) and atomic force microscope images (b) of H-terminated zigzag GNMs and the optical microscope image of the Hallmeasurement pattern on the fabricated GNM with an ionic-liquid gate (c), (d). The GNMs, which have honeycomb-like array of hexagonal nanopores, were fabricated on mechanically exfoliated graphenes via the nonlithographic method using a nano-porous alumina template (NPAT) as an etching mask [10,11].

The NPAT is easily fabricated through self-organization by anodic oxidation of pure (99.99\%) aluminum films. The graphene was carefully etched by optimized low-power Ar gas (e.g., 200 - $600 \mathrm{~V}$ for 10 - $40 \mathrm{~min}$ ) so as to avoid giving damages (Figure $\mathbf{1}(\mathrm{d})$ ) and the naomesh of the NPAT was transferred to graphene. Then, the NPAT was detached from the surface of the fabricated GNM, either mechanically or chemically.

The GNMs fabricated through these processes were annealed at a critical temperature $800^{\circ} \mathrm{C}$ in high vacuum ( $10^{-6}$ Torr) for 0.5 - 3 days with continual pumping of gas and, then, in hydrogen gas by the field-emission-type radical CVD system under pressure $>1 \mathrm{MPa}$ at least for 3 h. The first annealing is for deoxidization of the pore edges with recovering all damages and defects and is the key to forming zigzag pore edges by edge atomic reconstruction [10,12-14], while the second annealing is the key for termination of the carbon atoms at the pore edges by hydrogen atoms for production of flat-band ferromagnetism.

It is to be noted that for electrical measurements, we employed 5-layer graphenes, because monolayer GNMs exhibited poor electrical features. We have previously confirmed that even such thin-layer GNMs can exhibit ferromagnetism comparable with that of monolayer GNMs, although the amplitude reduces [1-3,22,23]. In fact, we have performed electrode fabrication and measurements 
after the confirmation of presence of the ferromagnetism.

The ionic-liquid gate was formed to apply $\mathrm{V}_{\text {ig }}$ following ref. [19] by dropping N, N-diethyl-N-(2-methoxyethyl)-N-methylammonium bis-(trifluoromethylsulfonyl)imide (DEME-TFSI) (Figures 1(c) and (d)). Applied $\mathrm{V}_{\mathrm{ig}}$ is consumed on at the interface of ionic liquid and the GNM, forming electrical-double layer within $\sim 1 \mathrm{~nm}$ thickness (Figure 1(d)). Thus, it induces extremely high electric fields on the GNM surface. In the present experiment, side-gate electrode not exposed to the ionic liquid was used to apply the $\mathrm{V}_{\mathrm{ex}}$.

\subsection{Changes in Electrical Properties and Extremely Large $\mathbf{I}_{\text {on/off }}$ upon $\mathbf{V}_{\text {ig }}$}

Figure 2 shows the drain current $\left(\mathrm{I}_{\mathrm{ds}}\right)$ as a function of the back-gate voltage $\left(\mathrm{V}_{\mathrm{bg}}\right)$ and $\mathrm{V}_{\mathrm{ig}}$ (i.e., before and after the formation of the ionic-liquid gate). Without the ionicliquid gate, the FM-GNM exhibits only n-type semiconductive behavior; $I_{d s}$ increases only with increasing $V_{b g}$ (Figure 2(a)). $I_{d s}$ is zero even when $V_{b g}$ is as small as $-20 \mathrm{~V}$. In contrast, with the ionic-liquid gating, $\mathrm{I}_{\mathrm{ds}}$ drastically increases with decreasing $V_{\text {ig }}$ (Figure 2(c)). Even when $V_{\text {ig }}$ is $\sim-1 \mathrm{~V}$, the p-type semiconductive behavior appears significantly. This can be attributed to hole doping by the partial oxygen-termination of the pore edges. Immediately before forming the ionic-liquid gate, the GNMs were exposed to an air atmosphere, because it is impossible to form the gate in high vacuum or a hydrogen atmosphere. Because the GNM is not covered by any passivation films, the pore edges are easily oxidized through this process, thereby resulting in the partial oxygen-termination and hole doping. Applying $-\mathrm{V}_{\text {ig }}$ significantly enhances this hole doping via pore edges. This occurrence is different from the case of bulk graphene without edges. Otherwise, the ionic-liquid gate itself might cause oxygen-like termination.

Figures 2(b) and (d) show the logarithmic scales of $I_{d s}$ transformed from Figures 2(a) and (c). The subthreshold slope values $\left(\mathrm{V}_{\text {sub }}\right)$ are $5 \mathrm{~V} /$ decade for increasing $\mathrm{I}_{\mathrm{ds}}$ in the $+\mathrm{V}_{\mathrm{bg}}$ region (Figure 2(b)) and $270 \mathrm{mV} /$ decade for increasing $\mathrm{I}_{\mathrm{ds}}$ in the $-\mathrm{V}_{\mathrm{ig}}$ region (Figure 2(d)). The $\mathrm{V}_{\text {sub }}$ value reflects the on-off ratio of $\mathrm{I}_{\mathrm{ds}}\left(\mathrm{I}_{\mathrm{on} / \mathrm{off}}\right)$ for the applied gate voltages and, hence, it is extremely important for field-effect transistor (FET) operation. The large $\mathrm{I}_{\text {on/off }}$ value directly leads to a high gain in the radio-frequency (RF) operation of FETs. The $\mathrm{V}_{\text {sub }}$ is inversely proportional to the $\mathrm{I}_{\mathrm{on} / \text { off. }}$. Previously, we have reported on the electrical characteristics of high-quality and low-defect GNRs derived from the unzipping of carbon nanotubes (CNTs) combined with three-step annealing [8]. These GNR-FETs exhibited a large $V_{\text {sub }}$ of $3 \mathrm{~V} /$ decade for a 20-nm width. The GNMs derived using polymer masks also exhibited a large $V_{\text {sub }}$ of $\sim 3 \mathrm{~V} /$ decade for a 7-nm

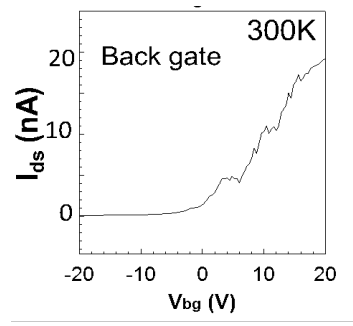

(a)

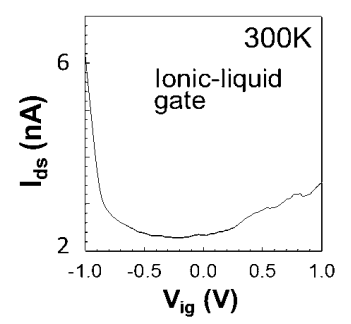

(c)

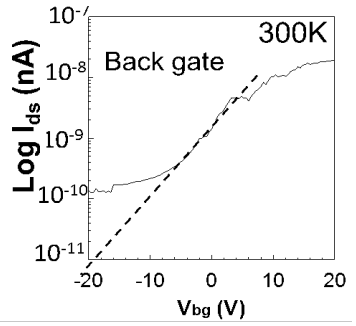

(b)

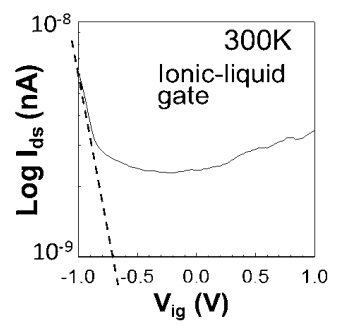

(d)
Figure 2. Electrical characteristics of GNM with back gate and ionic-liquid gate (a) Drain current $\left(\mathrm{I}_{\mathrm{ds}}\right)$ as a function of back-gate voltage $\left(\mathrm{V}_{\mathrm{bg}}\right)$ and $(\mathrm{b})$ its transformation to the logarithmic scale of $I_{d s}$ of the $\sim 5$-layer FM-GNMs. The GNMs show n-type semiconductive behavior and subthreshold slope values $\left(V_{\text {sub }}\right)$ of $5 \mathrm{~V} /$ decade. (c) $I_{d s}$ as a function of ionic-gate voltage $\left(V_{i g}\right)$ after formation of the ionic gate and (d) its transformation to the logarithmic scale of $I_{d s}$. A drastic increase in $I_{d s}$ in the $-V_{i g}$ region, indicative of p-type behavior, is observed with $V_{\text {sub }}$ as small as $\mathbf{2 7 0 ~} \mathrm{mV} /$ decade.

interpore width (i.e., GNR width) [29]. Only the GNRs obtained from the unzipping of CNTs exhibited a small $\mathrm{V}_{\text {sub }}$ of $210 \mathrm{mV} /$ decade but for a 2-nm width [9]. Thus, the present $V_{\text {sub }}$ of $270 \mathrm{mV} /$ decade for the 20 -nm interpore width is extremely small compared with those of any other GNR-FETs. This strongly suggests the effectiveness of ionic-liquid gating in producing and modulating extremely high electric fields on the GNM surface and the interpore GNR regions.

\subsection{Estimation of Carrier Densities by Magnetoresistance Observation and Large Geometrical Capacitance of Ionic-Liquid Gate}

We investigate the charge density $n_{s}$ achieved upon applying $V_{\text {ig }}$ by observing the magnetoresistance (MR) behaviors in the FM-GNMs.

When magnetic fields $(B)$ are applied perpendicular to the GNM plane, the electrons follow a cyclotron motion with the classical radius of the cyclotron orbit, $R_{c}=\left(\pi n_{s}\right)^{1 / 2} \hbar / e B$, where $h$ and $e$ denote Planck's constant and the electron charge, respectively [28]. A variety of interesting MR phenomena has been observed depending on the correlation of $2 R_{c}$ with $\phi$ (the pore diameter) and $a$ (the diameter of the unit cell). For instance, 
commensurability MR peaks and Aharonov-Bohm (AB)type oscillations (with an oscillation period

$B_{A B T}=(h / e) / S$, where $S$ denotes the area of the unit cell $)$ were observed around low $B$ values at points where electrons encircle and localize around the nanopores (see Figure 3(a); $2 R_{c}=a$ ) for samples with large $\phi / a$ values and small interpore spacing (i.e., $2(a-\phi))$ that is insufficient to allow electron cyclotron motion. In contrast, at high $B$ values, Shubnikov-de Haas (SDH) oscillations and consequent quantum Hall effects appeared for samples with small $\phi / a$ values and large interpore spacing that allowed for electron cyclotron motion with the small $R c$ values.

In a previous work on FM-GNMs with nearly identical structure parameters as those in the present one but without the ionic-liquid gate, a commensurability MR peak was observed at $B \sim 1.2 \mathrm{~T}$ [28]. From the expression $2 R_{c}=2\left(\pi n_{s}\right)^{1 / 2} \hbar / e B=a$, we estimated $n_{s} \approx 4 \times 10^{11} \mathrm{~cm}^{-2}$ and an elastic mean free path of $\ell_{e}=2 D / v_{F}$ (where $D$ denotes the diffusion constant and $v_{F}$ denotes the Fermi velocity) $\sim 800 \mathrm{~nm}$. Based on this result, we can estimate $n_{s}$ under extremely high electric fields caused by applying $V_{\text {ig }}$ to FM-GNMs with an ionic-liquid gate.

Figure 3 shows the MR $\left(R_{x x}\right)$ as a function of $V_{i g}$. The commensurability MR peak can be observed at $B \sim 1 \mathrm{~T}$ at $\mathrm{V}_{\mathrm{ig}}=0 \mathrm{~V}$. As $\mathrm{V}_{\mathrm{ig}}$ decreases, the peak shifts to larger $B$ regions. The maximum peak position of $B \sim 5 \mathrm{~T}$ is observed at $\mathrm{V}_{\mathrm{ig}}=-12 \mathrm{~V}$. This indicates an increase in $n_{s}$ with decreasing $\mathrm{V}_{\mathrm{ig}}$, because a large $n_{\mathrm{s}}$ value requires a large $B$ value in order to satisfy the condition for the commensurability peak: $2 R_{c}=a$. Consequently, the maximum $n_{s}$ of $\sim 1 \times 10^{13} \mathrm{~cm}^{-2}$ can be estimated from the peak $B$ value for $\mathrm{V}_{\text {ig }}=-12 \mathrm{~V}$.

This value is extremely large despite the small value of the assembled 20-nm-width GNR structure of the present GNM. This large value is attributed to the large electric field caused by $\mathrm{V}_{\text {ig. }}$. Moreover, this result also implies the contribution of the induced edge- $\pi$ states of the nanopores, in which high-density carriers localize at the pore edges even when $B=0$ (i.e., the large peak around $\mathrm{V}_{\mathrm{ig}}=$ $-12 \mathrm{~V}$ in Figure 4(b), explained later). These edge-localized carriers are added to the cyclotron elec- trons at $\mathrm{V}_{\mathrm{ig}}$ $=-12 \mathrm{~V}$, thereby resulting in extremely large values of $n_{s}$. Indeed, it is noteworthy that the peak position moves to a lower $B$ value and $n_{s}$ decreases at $V_{\text {ig }}=-16 \mathrm{~V}$. This evidences the contribution of the edge- $\pi$ electrons localized around the pore at $\mathrm{V}_{\mathrm{ig}}=-12 \mathrm{~V}$. Fur- thermore, the extremely large $n_{s}$ value results in a small mean free path $\left(l_{e}\right)$ of $\sim 800 \mathrm{~nm}$, which is considerably smaller than the circumferential length of the pore and cell $(\sim 250 \mathrm{~nm})$. Thus, the present GNM is not within a ballistic charge transport regime, and no AB-type oscillations are observed in Figure 3(b).

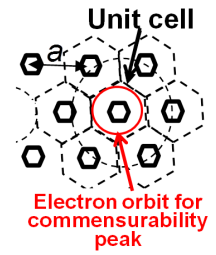

(a)

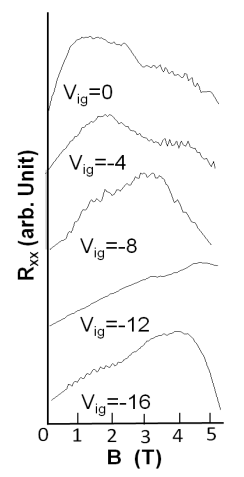

(b)

Figure 3. Magnetoresistance behavior for various ionicliquid gate voltages (a) Schematic view of an electron orbit for a commensurability MR peak in a GNM. The peak appears at a $B$ value where the electron cyclotron diameter $\left(2 R_{c}\right)$ is close to the unit cell diameter (a) and electrons localize in the unit cell. (b) MMR behavior $\left(R_{x x}\right)$ in a perpendicular field as a function of $V_{\text {ig }}$ of FM-GNMs with $~ 5$-layers at $T=1.5 \mathrm{~K}$. The broad MR peaks at each $V_{\text {ig }}$ value indicate the commensurability MR peaks mentioned in (a). Change in the peak position is caused by the modulation of $n_{s}$ upon changing $V_{\text {ig. }}$. The peak $B$ positions provide the $\boldsymbol{n}_{s}$ values of the FM-GNM at each $V_{i g}$, which includes the pore-edge localized electrons even for $B=0$.

In addition to the extremely high value of $n_{s}$, the other strong advantage of using the ionic-liquid gate is the obtainment of a large geometrical capacitance $\left(C_{\text {geo }}\right)$ [19], which allows the direct observation of the quantum capacitance $\left(C_{Q}\right)$. The large geometry of the ionic-liquid region (Figure 1(c)) provides an extremely large $C_{g e o}$. This capacitance allows the direct observation of the quantum capacitance $\left(C_{Q}=e^{2} D_{2 D}=m^{*} / \pi h^{2}\right.$, where $\mathrm{m}^{*}$ denotes the effective mass of carriers and $D_{2}$ is the electronic density of states of the $2 \mathrm{D}$ layer), because the total capacitance is given by $1 / C_{\text {tot }}=1 / C_{\text {geo }}+1 / C_{Q}$ and the $1 / C_{\text {geo }}$ value is negligible. In fact, a previous work has reported on the possible detection of $D_{2 D}$ at higher energy bands arising from the $C_{Q}$ of individual layers in monolayer to trilayer graphenes, using this method. Here, the capacitance of 2D systems is conventionally obtained from the Hall measurements (i.e., changes in $n_{s}$ as a function of $\left.V_{g}: C_{g e o}=e\left(\mathrm{~d} n_{s} / \mathrm{d} V_{g}\right)\right)$. However, it is difficult to employ this method in our case because the scattering by the honeycomb-like nanopore array prevents Hall measurements. 
Instead, we employ a GNR model in order to estimate $C_{g e o}$, and reconfirm the possibility of the direct observation of $C_{Q}$, because the interpore regions are GNRs (Figure 1). The GNR models gives carrier mobility

$\mu=g_{m} L / C_{g e o} V_{d s}$, where $g_{m}$ denotes the mutual conductance and $L$ denotes the GNR length8. The $g_{m}$ value of $\sim 500 \mathrm{mS}$ at $\mathrm{V}_{\mathrm{ds}}=0.8 \mathrm{~V}$ is observed in Figure 2(d), and $L$ corresponds to the inter-electrode distance of $2500 \mathrm{~nm}$ in our fabricated GNM. In a previous report, we have also estimated $\mu<\sim 100 \mathrm{~cm}^{2} / \mathrm{Vs}$ in FM-GNMs from MR measurements [28]. Consequently, $C_{g e o}$ for the side gate electrode is estimated to be $\sim 12 \mu \mathrm{F} / \mathrm{cm}^{2}$.

This value is in good agreement with those in previous reports on ionic-liquid (electrolytic) gates [19]. Conventional GNRs with widths of $2 \sim 20 \mathrm{~nm}$ placed on $\mathrm{SiO}_{2}$ substrates have $C_{\text {geo }} \sim 10^{-10}-10^{-12} \mathrm{~F}$ for the source electrodes. Thus, the present $C_{\text {geo }}$ value is nearly $10^{5}$ times greater than these reported values. Therefore, we can estimate $C_{Q}$ and $D_{2 D}$ directly from the measurement results related to $C_{\text {tot }}$ also in our GNM.

\subsection{Observation of Differential Conductivity on $\mathbf{V}_{\text {ig }}$}

Figure 4(a) shows the differential conductivity $(d I / d V$; $\mathrm{R}_{\mathrm{xx}}{ }^{-1}$ ) as a function of $\mathrm{V}_{\mathrm{ig}}$ in the low- $\mathrm{V}_{\mathrm{ig}}$ region when $B=$ 0 T. Even in the present $\sim$ 5-layer GNM, a Dirac-pointlike dip (zero-bias anomaly) is observed in the $\mathrm{d} I / \mathrm{d} V$ curves. Moreover, as the absolute value of $\mathrm{V}_{\mathrm{ig}}$ increases, other dips and peaks appear. Such $\mathrm{d} I / \mathrm{d} V$ anomalies due to $V_{\text {ig }}$ variation have been reported in tri-layer graphenes with ionic-liquid gates and attributed to carrier filling in higher energy bands (i.e., interband scattering). This is a advantage unique to the electrolytic gate method for the abovementioned reasons (i.e., introducing large electric fields and the presence of a large $C_{g e o}$ ). The curves in Figure 4(a) are qualitatively consistent with the abovementioned features. However, in Figure 4(a), the $\mathrm{d} I / \mathrm{d} V$ anomalies observed at $\mathrm{V}_{\mathrm{ig}} \sim \pm 0.3 \mathrm{~V}$ are considerably more defined than those observed in the tri-layer graphenes. Moreover, the plateau of the second peak in the $+\mathrm{V}_{\text {ig }}$ region is broad and consists of very small peaks. The presence of these peaks might suggest very strong interband scattering and the presence of a complicated higher-band structure in our 5-layer FM-GNM.

In the large $\mathrm{V}_{\mathrm{ig}}$ region (Figure 4(b)), we observe many $\mathrm{d} I / \mathrm{d} V$ peaks. In particular, a significantly large and broad $\mathrm{d} I / \mathrm{d} V$ peak is observable around $\mathrm{V}_{\mathrm{ig}} \sim-12 \mathrm{~V}$, when the side-gating $\mathrm{V}_{\mathrm{ex}}$ is $0 \mathrm{~V}$ (dotted line in Figure $4(\mathrm{~b})$ ). Although the $\mathrm{d} I / \mathrm{d} V$ properties as a whole are very complicated, the observed large $d I / d V$ values in the $-V_{\text {ig }}$ region at $\mathrm{V}_{\mathrm{ex}}=0 \mathrm{~V}$ are consistent with the hole-dominant property in Figures 2(c) and (d). This consistency indicates
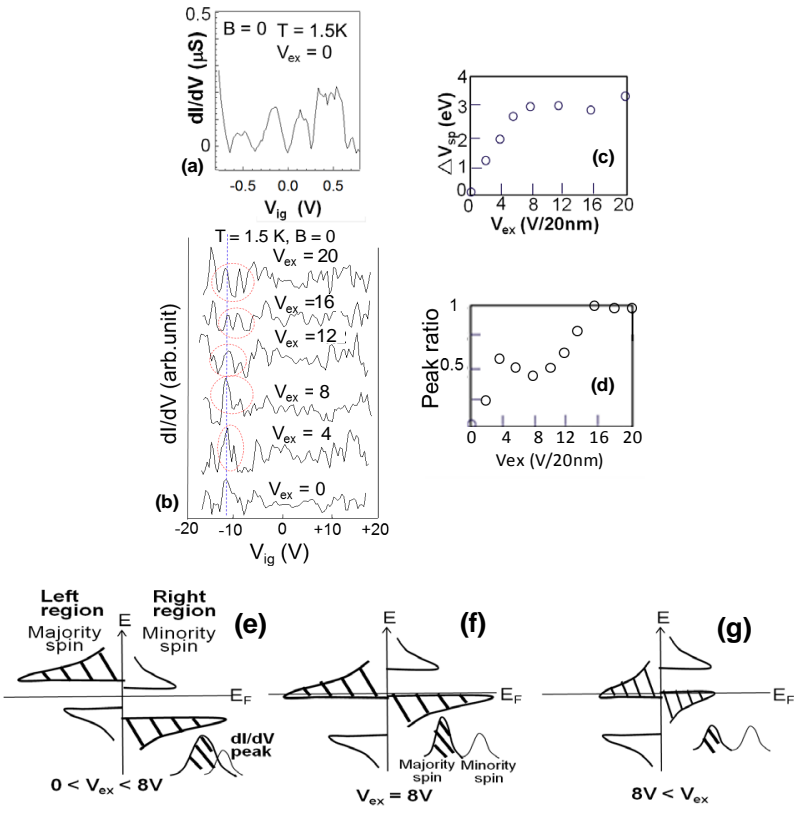

Figure 4. Conductivity behaviors for various ionic-liquid gate voltages $V_{\text {ig }}$ and side-gate (in-plane external) voltages $V_{\text {ex }}$ (a) Differential conductivity (dI/dV) as a function of $V_{i g}$ at low $V_{\text {ig }}$ values. (b) $d I / d V$ curves in the high- $V_{\text {ig }}$ regions with side-gating $V_{e x}$. The dashed vertical line indicates the location of the maximum peak and the dashed circle indicates its splitting into two peaks with increasing $V_{\mathrm{ex}}$. (c) The spin splitting voltage $\Delta V_{\text {ig }}$ estimated from the two peaks in Figure 4(b) as a function of side-gate $V_{\text {ex }}$. (d) Peak ratio for left/right peaks as a function of $V_{e x}$ in Figs.4b and 4c. It should be noticed that the peak ratio starts to significantly increase from the critical $V_{\mathrm{ex}} \sim 8 \mathrm{~V}$ due to the reduction in the height of the left peak, at which point $\Delta V_{\text {ig }}$ saturates in Figure 4(c). (e)-(g) Hal-metallicity-based schematics of changes in spin bands for majority and minority spins at both edges of a FM-GNR (corresponding to the interpore region) as a function of the in-plane $V_{\text {ex }}$. Insets: The schematics of the two dI/dV peaks shown in Figure 4(b).

the significant appearance of p-type semiconductive behavior.

The $\mathrm{d} I / \mathrm{d} V$ values typically have a strong correlation with $D_{2 D}$ and $n_{s}$, conventionally. A large $D_{2 D}$ value around Fermi level results in a large $\mathrm{d} I / \mathrm{d} V$ value $[16,17]$. Hence, the maximum $\mathrm{d} I / \mathrm{d} V$ peak around $\mathrm{V}_{\mathrm{ig}} \sim-12 \mathrm{~V}$ (Figure 4(b)) should reflect the maximum $D_{2 D}$ value of our FMGNM.

Because the edge-localized flat $\pi$ band has the largest $D_{2 D}$ values (edge states) and strongly contributes to the appearance of the ferromagnetism also in graphites [3, 21-23], the maximum $d I / d V$ peak should correspond to this edge- $\pi$ band. Theoretical works have proposed that the edge-magnetic moment arising from the edge- $\pi$ state survives even in graphites with interlayer interaction (e.g., ABA stacking with mono-hydrogenated edges) [3,21-23]. Moreover, because the present FM-GNM has only 5- 
layers, the influence of interlayer coupling for suppressing the emergence of ferromagnetism is very weak.

However, the localization of the edge-electrons prohibits current flow and obstructs detection of the large $D_{2 D}$ by $\mathrm{d} I / \mathrm{d} V$ observation in the present case, even if the $D_{2 D}$ value is very high. Nevertheless, we have reported certain MR behaviors (e.g., periodic MR oscillations [28] and saw-tooth-like MR oscillations [10]) originating from edge-localized $\pi$-electrons in similar $\sim 5$-layer FM-GNMs operating under a constant current mode in four-probe measurements. We have argued that certain numbers of pore-edge-localized electrons can travel between electrodes because of the constant current mode (i.e., under non-thermal equilibrium). Moreover, the $n_{\mathrm{s}}$ value of $\sim 1 \times$ $10^{13} \mathrm{~cm}^{-2}$ around $\mathrm{V}_{\mathrm{ig}} \sim-12 \mathrm{~V}$ observed in our ionic-liquid-gated FM-GNMs is considerably larger than the $n_{s}$ value of conventional FM-GNRs. This result supports the strong contribution of the drastically induced pore-edge $\pi$-electrons, as mentioned above. In such a case, the excess $\pi$-electrons localizing at the pore edges can be transported considerably more efficiently under a nonthermal equilibrium condition and contribute to the $\mathrm{d} I / \mathrm{d} V$. Therefore, we can assume that the maximum $\mathrm{d} I / \mathrm{d} V$ value directly reflects the edge-localized $\pi$ band with the highest DOS via the excess $\pi$ electrons, as seen from scanning tunnel microscopy observations of the DOS.

Such maximum $\mathrm{d} I / \mathrm{d} V$ peaks cannot be observed in conventional electrolytic-gated bulk graphenes without nanopores (i.e., without edge states), which showed no FM behavior, and such behavior has not thus far been observed in measurements obtained by applying $\mathrm{V}_{\mathrm{bg}}$. These results strongly support the correlation of the observed maximum $\mathrm{d} I / \mathrm{d} V$ peak with the induced pore-edge $\pi$ electrons in our ionic-liquid gated $\sim$ 5-layer FM-GNMs.

On the other hand, several other $\mathrm{d} I / \mathrm{d} V$ peaks are also observed in Figure 4(b). These should originate from other $D_{2 D}$ states, which are sensitive to spin interference by 1) the spin alignment of both the edges of the interpore GNR regions [3], 2) large ensemble of the GNR regions, 3) the interlayer stack structures [22], and also 4) $\mathrm{V}_{\mathrm{ig}}$ and $\mathrm{V}_{\mathrm{ex}}$. Thus, it is difficult to identify the origins of the individual peaks. For example, the second-largest peaks observed around $\mathrm{V}_{\mathrm{ig}} \sim-5 \mathrm{~V}$ and $-12 \mathrm{~V}$ at $\mathrm{V}_{\mathrm{ex}}=0$ in Figure 4(b) may be associated with the $\sigma$ band, because the present GNMs have $~ 5$-layer. In the present work, we focus on the largest $\mathrm{d} I / \mathrm{d} V$ peak observable around $\mathrm{V}_{\text {ig }} \sim-12 \mathrm{~V}$ at $\mathrm{V}_{\text {ex }}=0$ in Figure $4(\mathrm{~b})$, because Figures 4(b)-(d) suggest its strong correlation with the edge- $\pi$ band, as stated in next section.

\subsection{Splitting of a Maximum Conductance Peak by In-Plane External Fields and Half-Metallicity Model}

As mentioned above, we interestingly find that the maximum $\mathrm{d} I / \mathrm{d} V$ peak around $\mathrm{V}_{\mathrm{ig}} \sim-12 \mathrm{~V}$ separates into two peaks upon increasing the $\mathrm{V}_{\mathrm{ex}}$ (Figure 4(b)). The splitting voltage $\Delta \mathrm{V}_{\text {ig }}$ monotonically increases with increasing $\mathrm{V}_{\mathrm{ex}}$ and saturates at around $\mathrm{V}_{\mathrm{ex}}=8 \mathrm{~V}$ with $\Delta \mathrm{V}_{\mathrm{ig}}$ $\sim 3 \mathrm{eV}$ (Figure 4(c)). At low values of $\mathrm{V}_{\mathrm{ex}}$, the left peak is considerably more prominent than the right peak (Figure 4(b)), while the left peak height reduces for large $V_{e x}$ values, and eventually, the two peaks exhibit similar heights. The peak ratio for the left/right peaks after peak separation becomes equal with increasing $V_{e x}$ because of the decrease in the left-peak height (Figure 4(d)).

This spin splitting can be qualitatively understood by the resolving of the double spin degeneration of the edgelocalized flat $\pi$ band (i.e., separating of the majority- and minority-spin bands, which correspond to the left and right peaks, respectively) by applying the side-gating $\mathrm{V}_{\mathrm{ex}}$, which plays role like applying in-plane $\mathrm{V}_{\mathrm{ex}}$. Because the contribution of the localized minority-spins to $d / / d V$ is very small, the right-peak height is actually small throughout all the $\mathrm{V}_{\mathrm{ex}}$ regions (Figure 4(b)). Indeed, such a spin splitting upon the application of an electric field has been reported as an anomalous spin Hall effect in FM/non-FM hybrid materials (e.g., Pt/Ni-Fe). From the theoretical viewpoint, the presence of spin bands for the majority and minority spins has also been reported in FM-GNRs [3].

One particular theory [6] has predicted the half-metallicity and spin-gap opening that can be realized upon applying the in-plane field $\mathrm{V}_{\mathrm{ex}}$ in zigzag-GNRs with AF spin alignment at both the edges (as mentioned in the introduction). In our fabricated FM-GNMs, the spin alignment at the two edges of the interpore GNR region is FM. However, when a similar mechanism is realized for the majority and minority spins with opposite moments for FM-GNRs assembled between the electrodes, the above mentioned spin splitting can be observed (Figures 4(e)(g)), and it can be explained by assuming that the sidegate $\mathrm{V}_{\text {ex }}$ plays the role of the in-plane field $\mathrm{V}_{\mathrm{ex}}$. The predicted spin-gap opening as per the theory was $\Delta_{\text {theory }} \sim$ $0.5 \mathrm{eV}$ for $\mathrm{V}_{\mathrm{ex}}=0.1 \mathrm{~V} / \AA$. In contrast, the abovementioned value of $\Delta \mathrm{V}_{\mathrm{ig}} \sim 3 \mathrm{eV}$ for $\mathrm{V}_{\mathrm{ex}}=8 \mathrm{~V}$ corresponds to a considerably larger $\Delta \mathrm{V}_{\text {ig. }}$. As mentioned above, we have used the side-gating voltage $V_{e x}$ for this observation. When $\mathrm{V}_{\mathrm{ex}}=8 \mathrm{~V}$ is applied from the side-gate electrode directly placed on the Si substrate, the voltage propagates via the substrate surface, thereby leading to a potential difference between both sides of the individual interpore GNR with 20-nm width. The potential difference can be estimated to be $\sim 0.1 \mathrm{~V} / 20 \mathrm{~nm}$, based on our previous work [8]. Thus, $\Delta \mathrm{V}_{\mathrm{ig}} \sim 3 \mathrm{eV}$ for $\mathrm{V}_{\mathrm{ex}}=8 \mathrm{~V}$ corresponds to $\Delta \mathrm{V}_{\mathrm{ig}} \sim 600 \mathrm{eV}$ for $\mathrm{V}_{\mathrm{ex}}=0.1 \mathrm{~V} / \AA$. This $\Delta \mathrm{V}_{\mathrm{ig}}$ value is considerably larger than the theoretical prediction $\Delta_{\text {theory }} \sim$ $0.5 \mathrm{eV}$. 
However, because this value of $\Delta \mathrm{V}_{\mathrm{ig}} \sim 600 \mathrm{eV}$ is not the actual spin splitting energy in the GNM but corresponds to the $V_{\mathrm{ig}}$ value applied to the side-gate electrode, the actual splitting energy $\Delta V_{s p}$ needs to be calculated. The $\Delta \mathrm{V}_{\mathrm{sp}}$ value corresponds to the energy in the singleparticle energy spectrum given by

$$
\Delta V_{\text {sp }}=\hbar v_{F} \sqrt{2 \pi C_{\text {geo }} \Delta V_{\text {ig }} /|e|}
$$

Here, $v_{F} \sim 10^{3} \mathrm{~m} / \mathrm{s}$ denotes the Fermi velocity in our fabricated GNM, which is at least 1000 times smaller than that for bulk graphene [8] considering edge scattering. Using the previously estimated value of $C_{\text {geo }} \sim 12$ $\mu \mathrm{F} / \mathrm{cm}^{2}, \Delta \mathrm{V}_{\text {sp }}$ can be estimated to be $\sim 0.54 \mathrm{eV}$. This value is quantitatively in good agreement with the abovementioned theoretical prediction of $\Delta \mathrm{V}_{\text {theory }} \sim 0.5$ $\mathrm{eV}$. This estimation also implies that the edge-localized $\pi$ band (i.e., the observed $\mathrm{d} I / \mathrm{d} V$ peak at $\mathrm{V}_{\mathrm{ig}} \sim-12 \mathrm{~V}$ ) exists at $\sim 1.2 \mathrm{eV}$ below the initial Fermi level position at $\mathrm{V}_{\mathrm{ig}}=$ $0 \mathrm{~V}$. Because the FM-GNM consists of the assembled FM-GNRs, this might smear the $\mathrm{d} I / \mathrm{d} V$ maximum and also its splitting by averaging. However, we assume that only the dominant some GNRs (interpore regions), of which the width-direction (i.e., both edges) locates in parallel with electrodes to apply $V_{\text {ex }}$, and have perfect zigzag edges, strongly contribute to these phenomena.

Moreover, it should be noticed that the peak ratio starts to significantly increase from the critical $V_{\text {ex }}$ value of $8 \mathrm{~V}$ due to the reduction in the height of the left peak (Figure $4(\mathrm{~d})$ ), at which point $\Delta \mathrm{V}_{\text {ig }}$ saturates (Figure $4(\mathrm{c})$ ). This result supports the conclusions of the half-metallicity model [6]. Following this model, the saturation of $\Delta \mathrm{V}_{\mathrm{ig}}$ corresponds to the gap closing for one-moment spins existing at both edges of the GNRs (Figure 4(f)). Although this gap closing facilitates the same-moment spin current flow across the GNR, the localization of the edge electrons for the majority spin is suppressed by the spin current flow. A further increase in $\mathrm{V}_{\mathrm{ex}}$ results in a decrease in the amplitude of the edge states (i.e., resulting in a decrease in the excess $n_{s}$ ) for the same-moment spins and, thus, the left-peak height decreases (Figure 4(g)) despite appearance of the current flow.

Consequently, we suggest that the applied side-gate $\mathrm{V}_{\mathrm{ex}}$ causes spin splitting of the edge- $\pi$ band (for the majority and minority spins with opposite moments for FMGNRs) similar to that caused by the application of an inplane $\mathrm{V}_{\text {ex }}$ following a theory [6]. This is because extremely large values of the $D_{2 D}$, which originate from the edge states of the nanopores induced by $\mathrm{V}_{\mathrm{ig}}$, are highly sensitive to added external fields. In fact, we could observe no such $V_{e x}$-dependent changes in the electronic states in the absence of $\mathrm{V}_{\text {ig }}$ (in this case, we applied only the back-gate voltage and, consequently, we could not detect even edge- $\pi$ states, as in Figure 4). Because the present GNM is placed on a $\mathrm{SiO}_{2}$ film, the side-gating $\mathrm{V}_{\mathrm{ex}}$ cannot directly modulate the interpore GNR edges. However, when the ionic-liquid gate is formed, the liquid faces the pore edges (i.e., both edges of an interpore GNR) and also the surface of $\mathrm{SiO}_{2}$ at the pore's inner regions. We speculate that such conditions drastically change the large edge-electronic states even for a small potential difference existing between the two edges of the interpore GNR region caused by the side-gating $\mathrm{V}_{\mathrm{ex}}$, and the $V_{\text {ex }}$ acts in a manner similar to the actual in-plane field $V_{\text {ex }}$.

\subsection{Rapid Degradation of Samples during Electrical Measurements}

Subsequent to measurements performed over nearly three days, we observed a degradation of the electrical and magnetic characteristics of the FM-GNM. In particular, the appearance of single-electron charging effect was observed in the current channel region (Figure 5).

The staircase-like feature is confirmed in the Ids vs. $\mathrm{V}_{\mathrm{ds}}$ relationship in Figure 5. The inset shows $\mathrm{dI}_{\mathrm{ds}} / \mathrm{dV}_{\mathrm{ds}}$ as a function of $\mathrm{V}_{\mathrm{ds}}$. It exhibits a periodical oscillation with a period of $180 \mathrm{mV}$. It is well known that such a phenomenon is understood as the Coulomb staircase, which originates from the charging effect of a single electron in an extremely small dot area with a charging energy of $E_{c}$ $=\mathrm{e}^{2} / 2 \mathrm{C}_{\text {dot }} \sim 180 \mathrm{meV}$, where $\mathrm{C}_{\text {dot }}$ denotes the capacitance of the dot area $[30,31]$. The $\mathrm{C}_{\mathrm{dot}}$ value corresponds to $\sim 10^{-18} \mathrm{~F}$, which is a value that is considerably smaller than $C_{g e o}$ and also any other capacitances of the present GNM-FET structure. This result strongly suggests the emergence of small defects in the current channel region due to degradation after the three-day measurements. Indeed, ref. [16] reported on the quick degradation of graphenes with electrolytic gates and the consequent importance of obtaining quick measurements.

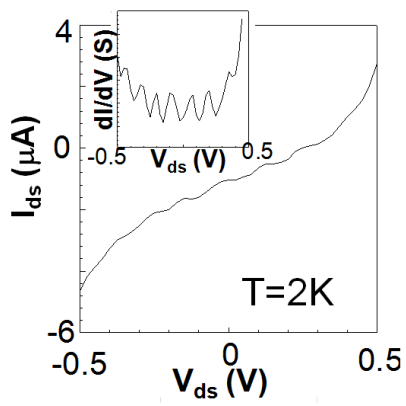

Figure 5. Electrical characteristics after three-day measurements $I_{d s}-V_{d s}$ relationship after the measurements through nearly three days. The sample was placed in a He-gas atmosphere in a Cryomag system (Oxford Inst.) at $\mathrm{T}=1.5 \mathrm{~K}$ during the measurements. Inset: $\mathrm{dI}_{\mathrm{ds}} / \mathrm{dV}_{\mathrm{ds}}$ as a function of $V_{d s}$. The curve exhibits a periodical oscillation with a period of $180 \mathrm{mV}$, the so-called Coulomb staircase. 
The sample was placed under high-vacuum conditions in a Cryomag system (Oxford Instruments) at $\mathrm{T}=1.5 \mathrm{~K}$ during the measurements. Thus, we concluded that the edge degradation is due to the chemical reaction of the (frozen) ionic liquid with the GNM during the measurement. Because the $\mathrm{H}$-terminated pore edges face the ionic liquid, they are most sensitive to such degradation. In the present edge-related measurements, a more careful treatment of the GNMs will be required, because it is well known that the edge-based phenomena are highly sensitive to edge degradation.

Moreover, in our previous works, we have reported observing AB-type MR oscillations [28] and saw-tooth like MR oscillations (i.e., the spin pumping effect [10]) arising from the localized electrons at the pore edges in 5-layer FM-GNMs without ionic-liquid gating. On the other hand, in the present structure with ionic-liquid gates, these oscillations are hardly observed. This absence of oscillations might also be due to the partial degradation caused by the chemical reaction between the pore-edge carbon atoms and the ionic liquid.

\section{Conclusions}

Graphene edges with a zigzag-type atomic structure can theoretically produce spontaneous spin polarization despite being a critical-metal-free material. We previously demonstrated this in GNMs with honeycomb-like arrays of low-defect hexagonal nanopores by observing room temperature ferromagnetism and spin-based phenomena arising from the zigzag-pore edges.

In the present work, we applied extremely high electric fields to the FM-GNMs using an ionic-liquid gate. A large on/off-ratio for hole current was observed for even small applied $\mathrm{V}_{\text {ig. }}$. Observations of the MR behavior revealed the carrier densities as high as $\sim 10^{13} \mathrm{~cm}^{-2}$ under

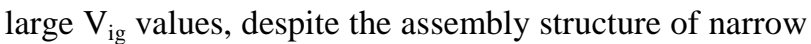
GNRs with $\sim 20 \mathrm{~nm}$ width. $C_{\text {geo }}$ as large as $\sim 12 \mu \mathrm{F} / \mathrm{cm}^{2}$ was also estimated. We observed a maximum conductance peak in the high $-\mathrm{V}_{\text {ig }}$ region and its separation into two peaks upon applying a side-gate $\mathrm{V}_{\mathrm{ex}}$. Based on halfmetallicity model, it was discussed that the localized edge- $\pi$ band with excess-density electrons induced by $\mathrm{V}_{\mathrm{ig}}$ and its spin splitting for majority and minority spins caused by $V_{\mathrm{ex}}$ led to these phenomena and that the applied $\mathrm{V}_{\text {ex }}$ played a role similar to that of the in-plane field $\mathrm{V}_{\mathrm{ex}}$ under the application of $\mathrm{V}_{\mathrm{ig}}$.

The present finding clarify that one can detect the localized edge bands via the excess-density electrons due to $V_{\text {ig }}$ by observing conductance and also the spin behaviors of the band within controlled manner by applying side-gate $\mathrm{V}_{\mathrm{ex}}$. The results must be valuable for criticalelement-free novel spintronic devices.

\section{Acknowledgements}

The authors thank Y. Iwasa, K. Fujita, Y. Hashimoto, E. Endo, Y. Iye, S. Katsumoto, M. Yamamoto, S. Tarucha for their technical contribution, fruitful discussions, and encouragement. This work was partly supported by a Grant-in-Aid for Scientific Research (Basic research A: 24241046) under the aegis of MEXT, a Grant for Zeroemission Energy program by Kyoto University, and also an AFOSR grant.

\section{REFERENCES}

[1] K. Nakada, M. Fujita, G. Dresselhaus and M. S. Dresselhaus, "Edge State in Graphene Ribbons: Nanometer Size Effect and Edge Shape Depend,” Physical Review B, Vol. 54, No. 24, 1996, pp. 17954-17961. http://dx.doi.org/10.1103/PhysRevB.54.17954

[2] M. Fujita, K. Wakabayashi, K. Nakada and K. Kusakabe, "Peculiar Localized State at Zigzag Graphite Edge," Journal of the Physical Society of Japan, Vol. 65, 1996, pp. 1920-1923. http://dx.doi.org/10.1143/JPSJ.65.1920

[3] H. Lee, Y. Son, N. Park, S. Han and J. Yu, "Magnetic Ordering at the Edges of Graphitic Fragments: Magnetic Tail Interactions between the Edge-Localized States," Physical Review B, Vol. 72, No. 17, 2005, Article ID: 174431. http://dx.doi.org/10.1103/PhysRevB.72.174431

[4] R. G. A. Veiga, R. H. Miwa and G. P. Srivastava, "Quenching of Local Magnetic Moment in Oxygen Adsorbed Graphene Nanoribbons," The Journal of Chemical Physics, Vol. 128, 2008, Article ID: 201101. http://dx.doi.org/10.1063/1.2937453

[5] T. Enoki and K Takai, "The Edge State of Nanographene and the Magnetism of the Edge-State Spins," Solid State Communications, Vol. 149, No. 27-28, 2009, pp. 11441150. http://dx.doi.org/10.1016/j.ssc.2009.02.054

[6] Y. W. Son, M. L. Cohen and S. G. Louie, "Half-Metallic Graphene Nanoribbons,” Nature, Vol. 444, 2006, pp. 347-349. http://dx.doi.org/10.1038/nature05180

[7] L. Yang, C. Park, Y. Son, M. L. Cohen and S. G. Louie, "Quasiparticle Energies and Band Gaps in Graphene Nanoribbons,” Physical Review Letters, Vol. 99, No. 18, 2007, Article ID: 186801. http://dx.doi.org/10.1103/PhysRevLett.99.186801

[8] T. Shimizu, J. Haruyama, D. C. Marcano, D. V. Kosinkin, J. M. Tour, K. Hirose and K. Suenaga, "Large Intrinsic Energy Bandgaps in Annealed Nanotube-Derived Graphene Nanoribbons," Nature Nanotechnology, Vol. 6, 2011, pp. 45-50.

http://dx.doi.org/10.1038/nnano.2010.249

[9] X. Wang, Y. Ouyang, X. Li, H. Wang, J. Guo and H. Dai, "Room-Temperature All-Semiconducting Sub-10-nm Graphene Nanoribbon Field-Effect Transistors,” Physical Review Letters, Vol. 100, No. 20, 2008, Article ID: 206803. http://dx.doi.org/10.1103/PhysRevLett.100.206803

[10] K. Tada, T. Hashimoto, J. Haruyama, H. Yang and M. Chshiev, "Spontaneous Spin Polarization and Spin Pum- 
ping Effect on Edges of Graphene Antidot Lattices," Solid State Physics, Vol. 249, No. 12, 2012, pp. 24912496. http://dx.doi.org/10.1002/pssb.201200042

[11] I. Takesue, J. Haruyama, N. Kobayashi, S. Chiashi, S. Maruyama, T. Sugai and H. Shinohara, "Superconductivity in Entirely End-Bonded Multiwalled Carbon Nanotubes,” Physical Review Letters, Vol. 96, No. 5, 2006, Article ID: 057001. http://dx.doi.org/10.1103/PhysRevLett.96.057001

[12] X. Jia, M. Hofmann, V. Meunier, B. G. Sumpter, J. Campos-Delgado, J. M. Romo-Herrera, H. Son, Y. Hsieh, A. Reina, J. Kong and M. S. Dresselhaus, "Controlled Formation of Sharp Zigzag and Armchair Edges in Graphitic Nanoribbons," Science, Vol. 323, No. 5922, 2009, pp. 1701-1705. http://dx.doi.org/10.1126/science.1166862

[13] Ç. Ö. Girit, J. C. Meyer, R. Erni, M. D. Rossell, C. Kisielowski, L. Yang, C. Park, M. F. Crommie, M. L. Cohen and S. G. Louie, "Graphene at the Edge: Stability and Dynamics,” Science, Vol. 323, No. 5922, 2009, pp. 17051708. http://dx.doi.org/10.1126/science.1166999

[14] Y. M. You, Z. H. Ni, T. Yu and Z. X. Shen, "Edge Chirality Determination of Graphene by Raman Spectroscopy,” Applied Physics Letters, Vol. 93, No. 16, 2008, Article ID: 163112. http://dx.doi.org/10.1063/1.3005599

[15] B. Krauss, P. Nemes-Incze, V. Skakalova, L. P. Biro, K. von Klitzing and J. H. Smet, "Raman Scattering at Pure Graphene Zigzag Edges,” Nano Letters, Vol. 10, No. 11, 2010, pp. 4544-4548. http://dx.doi.org/10.1021/nl102526s

[16] D. K. Efetov and P. Kim, "Controlling Electron-Phonon Interactions in Graphene at Ultrahigh Carrier Densities," Physical Review Letters, Vol. 105, No. 25, 2010, Article ID: 256805.

http://dx.doi.org/10.1103/PhysRevLett.105.256805

[17] D. K. Efetov, P. Maher, S. Glinskis and P. Kim, "Multiband Transport in Bilayer Graphene at High Carrier Densities,” Physical Review B, Vol. 84, No. 16, 2011, Article ID: 161412(R).

[18] A. Das, A. K. Geim, et al., "Monitoring Dopants by Raman Scattering in an Electrochemically Top-Gated Graphene Transistor," Nature Nanotechnology, Vol. 3, 2008, p. 210. http://dx.doi.org/10.1038/nnano.2008.67

[19] M. Panzer, et al., "Photo-Embossed Surface Relief Structures with an Increased Aspect Ratios by Addition of a Reversible Addition-Fragmentation Chain Transfer Agent," Advanced Materials, Vol. 20, No. 16, 2008, p. 3117. http://dx.doi.org/10.1002/adma.200800124

[20] K. F. Mac, et al., "Observation of an Electric-Field-Induced Band Gap in Bilayer Graphene by Infrared Spectroscopy,” Physical Review Letters, Vol. 102, No. 25, 2009, Article ID: 256405.
http://dx.doi.org/10.1103/PhysRevLett.102.256405

[21] A. H. R. Pasler, "Interlayer Interactions in Graphite and Carbon Nanotubes,” Chemical Physics, Vol. 1, No. 18, 1999, p. 4459.

[22] M. Otani, M. Koshino, Y. Takagi and S. Okada, "Intrinsic Magnetic Moment on (0001) Surfaces of Rhombohedral Graphitee,” Physical Review B, Vol. 81, No. 16, 2010, Article ID: 161403 (R). http://dx.doi.org/10.1103/PhysRevB.81.161403

[23] M. Otani, Y. Takagi, M. Koshino and S. Okada, "Phase Control of Magnetic State of Graphite Thin Films by Electric Field,” Appl. Phys. Lett., Vol. 96, No. 24, 2010, pp. 242504.http://dx.doi.org/10.1063/1.3455069

[24] S. Murakami, N. Nagaosa and S. Zhang, "Dissipationless Quantum Spin Current at Room Temperature,” Science, Vol. 301, No. 5638, 2003, pp. 1348-1351. http://dx.doi.org/10.1126/science.1087128

[25] C. L. Kane and E. J. Mele, "Quantum Spin Hall Effect in Graphene,” Physical Review Letters, Vol. 95, No. 22, 2005, pp. 226801-226804. http://dx.doi.org/10.1103/PhysRevLett.95.226801

[26] M. J. Schmidt and D. Loss, "Edge States and Enhanced Spin-Orbit Interaction at Graphene/Graphane Interfaces," Physical Review B, Vol. 81, No. 16, 2010, Article ID: 165439. http://dx.doi.org/10.1103/PhysRevB.81.165439

[27] D. A. Abanin, S. V. Morozov, L. A. Ponomarenko, R. V. Gorbachev, A. S. Mayorov, M. I. Katsnelson, K. Watanabe, T. Taniguchi, K. S. Novoselov and L. S. Levitov, "Giant Nonlocality near the Dirac Point in Graphene," Science, Vol. 332, No. 6027, 2011, pp. 328-330. http://dx.doi.org/10.1126/science.1199595

[28] T. Shimizu, J. Nakamura, K. Tada, Y. Yagi and J. Haruyama, "Magnetoresistance Oscillations Arising from Edge-Localized Electrons in Low-Defect Graphene Antidot-Lattices," Applied Physics Letters, Vol. 100, No. 2, 2012, Article ID: 023104. http://dx.doi.org/10.1063/1.3675547

[29] J. Bai, X. Zhong, S. Jiang, Y. Huang and X. Duan, “Graphene Nanomesh,” Nature Nanotechnology, Vol. 5, 2010, pp. 190-194. http://dx.doi.org/10.1038/nnano.2010.8

[30] H. Grabert and M. H. Devoret, "Single Charge Tunneling," NATO ASI Series B, Vol. 294, Plenum, New York, 1991.

[31] J. Haruyama I. Takesue, T. Hasegawa and Y. Sato, “Coulomb Blockade Related to a Localization Effect in a Single Tunnel-Junction/Carbon-Nanotube System,” Physical Review B, Vol. 63, No. 7, 2001, Article ID: 073406. http://dx.doi.org/10.1103/PhysRevB.63.073406 